\title{
Observing Mercury: from Galileo to the stereo camera on the BepiColombo mission
}

\author{
Gabriele Cremonese $^{1}$, Vania Da Deppo ${ }^{2}$, Giampiero Naletto ${ }^{2,3,4}$, \\ Elena Martellato ${ }^{4}$, Stefano Debei ${ }^{4}$, Cesare Barbieri ${ }^{4,5}$, Carlo \\ Bettanini $^{4}$, Maria T. Capria ${ }^{6}$, Matteo Massironi ${ }^{7}$, Mirko Zaccariotto ${ }^{4}$ \\ ${ }^{1}$ INAF - Osservatorio Astronomico, \\ Vic. Osservatorio 5, I-35122 Padova, Italy \\ email: gabriele.cremonese@oapd.inaf.it \\ ${ }^{2}$ CNR-IFN UOS Luxor, Padova, Italy \\ email: dadeppo@dei.unipd.it \\ ${ }^{3}$ Dep. of Information Engeneering, University of Padova, \\ email: naletto@dei.unipd.it \\ ${ }^{4}$ CISAS, University of Padova, \\ Via Venezia 15, 35131, Padova, Italy \\ email: elena.martellato@oapd.inaf.it \\ email: stefano.debei@unipd.it \\ ${ }^{5}$ Astronomy Department, University of Padova, \\ Vic. Osservatorio 3, 35122, Padova, Italy \\ email: cesare.barbieri@unipd.it \\ ${ }^{6}$ INAF - IASF, Via del Fosso del Cavaliere 10000133 Roma, Italy \\ email: mariateresa.capria@iasf-roma.inaf.it \\ ${ }^{7}$ Dep.Geosciences, University of Padova, Via Giotto, 35137 Padova, Italy \\ email: matteo.massironi@unipd.it
}

\begin{abstract}
After having observed the planets from his house in Padova using his telescope, in January 1611 Galileo wrote to Giuliano de Medici that Venus is moving around the Sun as Mercury. Forty years ago, Giuseppe Colombo, professor of Celestial Mechanics in Padova, made a decisive step to clarify the rotational period of Mercury. Today, scientists and engineers of the Astronomical Observatory of Padova and of the University of Padova, reunited in the Center for Space Studies and Activities (CISAS) named after Giuseppe Colombo, are busy to realize a stereo camera (STC) that will be on board the European (ESA) and Japanese (JAXA) space mission BepiColombo, devoted to the observation and exploration of the innermost planet. This paper will describe the stereo camera, which is one of the channels of the SIMBIOSYS instrument, aiming to produce the global mapping of the surface with $3 \mathrm{D}$ images.
\end{abstract}

Keywords. space vehicles: instruments, planets: Mercury

\section{Introduction}

The city and the University of Padova have a long standing love affair with the inner planets, Mercury in our case, since those very momentous days of fall - winter 1609 crowned by the Sidereus Nuncius, when Galileo Galilei made some of his most important discoveries using his 'cannocchiale'. Shortly after, in January 1611, he wrote to Giuliano de Medici stating that Venus and Mercury move around the Sun as the other planets do. Around the middle of the XX Century, Giuseppe Colombo, professor of Celestial Mechanics in the same University, made a decisive step to clarify the relationships 
between orbit and spin of the planet. To acknowledge such important role, the European Space Agency has named BepiColombo the forthcoming mission to Mercury devoted to the observation and exploration of the innermost planet. The names of Galileo and Colombo are thus associated to two of the most important space missions in the solar system. Today, scientists and engineers of the Astronomical Observatory and of the University of Padova, reunited in the Center for Space Studies and Activities (CISAS) named after Giuseppe Colombo, are working on a stereo camera (STC) that will be on board BepiColombo. This paper will describe the stereo camera (STC), which is one of the channels of the SIMBIOSYS instrument, aiming to produce the global mapping of the surface in $3 \mathrm{D}$.

\section{SIMBIOSYS}

The Spectrometer and Imagers for MPO BepiColombo Integrated Observatory SYStem (SIMBIOSYS) (Figure 1) will be on board the Mercury Planetary Orbiter of the ESA-JAXA mission BepiColombo (Flamini et al. 2010). SIMBIOSYS includes a high resolution imaging channel (HRIC), providing images at a spatial resolution of $5 \mathrm{~m} /$ pixel at the periherm, the VIS-NIR spectrometer (VIHI) that will provide global mapping of Mercury's surface in the spectral range $400-2200 \mathrm{~nm}$, with a spectral sampling of $6.25 \mathrm{~nm}$, and a spatial resolution of $400 \mathrm{~m} /$ pixel at the periherm, and the stereo imaging channel (STC) (Cremonese et al. 2009) that will be described in the following sections.

\section{General Scientific Objectives of STC}

The analysis of the geological characteristics of Mercury's surface requires the mapping, at a resolution lower than 110 m per pixel, and the Digital Terrain Model (DTM) of the entire surface. The main scientific objectives can be summarized as follows:

- large scale Mercury's surface composition. The STC filters are located in the spectral range of 400-920 $\mathrm{nm}$ where the electronic processes in the transition elements give the major contribution to the absorption spectra of minerals. The color mapping of the STC will therefore allow to discriminate among rock-forming minerals primarily on the basis of the albedo and color contrasts;

- Mercury's lithostratigraphic units identification, as smooth plains, intercrater plains, heavily cratered terrains and hilly and lineated terrains. STC will constrain the relationship between different geological units in extended regions; therefore, a satisfactory knowledge of the global stratigraphy will be achieved;

- cratering record and surface age, which is an outstanding tool for geological dating and provides important information on the origin of impacting objects;

- volcanism. STC accuracy on 3D reconstruction will allow the detection of several volcanic structures and deposits (Massironi et al. 2008) that can be described in three main objectives:

o identification of different lava flows and volcanic deposits with definition of their emplacement mechanisms;

○ identification of volcanic edifices, domes and dykes;

- identification of possible fissured vents.

- tectonic. The 3D restitution obtained by STC images should lead to a global mapping only slightly affected by directional interpretation errors since the derived DTM will reflect more directly the morphological pattern of the surface. Therefore, the despinning effect, cooling or dynamical loading that changed the planet shape during the early history can be better distinguished and quantitatively constrained. 


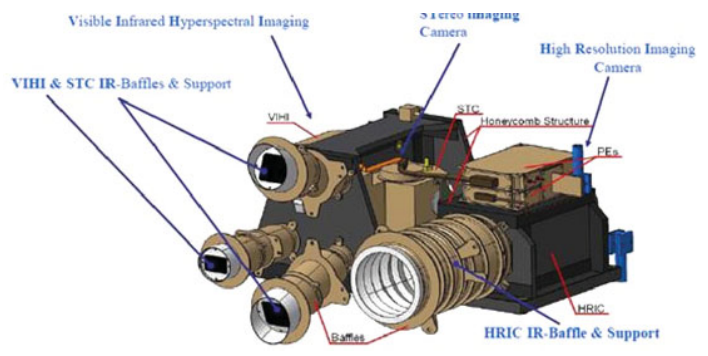

Figure 1. The SIMBIOSYS suite

Table 1. Summary of the STC nominal performance.

\begin{tabular}{ll}
\hline Parameter & Value \\
\hline Scale factor & $50 \mathrm{~m} /$ pix at periherm \\
Swath & $38 \mathrm{~km}$ at periherm \\
Stereoscopic properties & \pm 20 stereo angle with respect to nadir both images \\
& on the same detector \\
Vertical accuracy & $80 \mathrm{~m}$ \\
EE & $\geqslant 70 \%$ inside 1 pix \\
MTF & $\geqslant 60 \%$ at Nyquist frequency \\
Wavelength coverage & $410-930 \mathrm{~nm}(5$ filters $)$ \\
Filters & Panchromatic $(700 \pm 100 \mathrm{~nm})$ \\
& $420 \pm 10 \mathrm{~nm}$ \\
& $550 \pm 10 \mathrm{~nm}$ \\
& $750 \pm 10 \mathrm{~nm}$ \\
& $920 \pm 10 \mathrm{~nm}$ \\
\hline
\end{tabular}

Table 2. Summary of the STC optical parameters

\begin{tabular}{ll}
\hline Parameter & Value \\
\hline Optical concept & modified Schmidt telescope \\
& with rhomboid prisms and field corrector \\
2 identical optical sub-channels; \\
detector and most of the optical elements \\
common to the two sub-channels
\end{tabular}

\section{STC Optical Design}

The stereo camera for the BepiColombo mission is based on a completely new optical design and acquisition technique, as the push-frame, instead of the push-broom techniques used by HRSC on board the Mars Express mission and the terrestrial satellites SPOT. We think that the push-frame technique allow us to achieve optimal results reducing the number of images to mosaic and having larger single images to find the matching points for the stereo reconstruction. The global FoV of each channel is $5.3^{\circ} \times 4.5^{\circ}$, 
subdivided in 3 strips, one for each filter, covering 3 quasi-contiguous strips on Mercury surface; at periherm, on the equator, each strip corresponds to an area of about $38 \times 19 \mathrm{~km}^{2}$ for the panchromatic filter and of about $38 \times 3 \mathrm{~km}^{2}$ for the color ones (Figure 2).

The design has been kept as short and compact as possible, with the need of having the possibility to baffle the two channels to cope with straylight problems due to the common optics. The desired $50 \mathrm{~m} / \mathrm{px}$ scale factor at periherm is achieved with a 90 $\mathrm{mm}$ system effective focal length, considering the choice of a $10 \mu \mathrm{m}$ pixel size hybrid SiPIN CMOS as detector. This kind of detector is particularly useful both in terms of radiation hardness, given the hostile Mercury environment, and for the capability of snapshot image acquisition, allowing very short exposure times of about $1 \mathrm{~ms}$ for STC. The optical sub-system consists of a modified Schmidt telescope plus a couple of rhomboid prisms positioned in front of the objective. In that solution, the classical Schmidt correcting plate positioned in the center of the curvature of the spherical mirror has been substituted with a correcting doublet positioned at about half distance: in this way the volume is reduced of about a factor of two with respect to the classical solution (Figure 3). All the optical elements, except the rhomboid prisms and the aperture stops (AS), are common to both channels. Common optics, together with the fact that the rhomboid prisms are not sensitive to the tilt, make the system free of co-registration error between the channels. Moreover, to optimize the separation between the optical paths of the two channels, an ad hoc baffling system is proposed and designed.

Table 1 and 2 summarize the optical characteristics of STC and its nominal performance respectively.

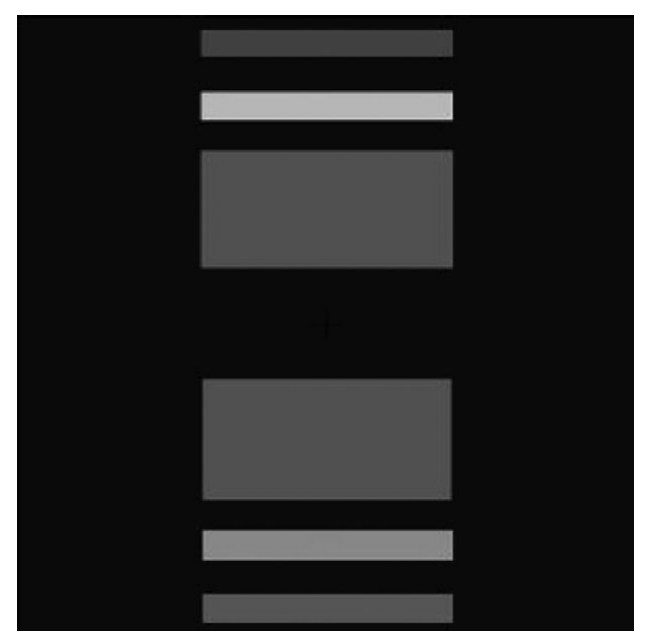

Figure 2. Position and size of the filter strips (depicted in light gray) on the full $2 \mathrm{kx} 2 \mathrm{k}$ detector area (in black).

\section{STC Mechanical Design}

STC is mainly composed by 3 sub-units:

- focal Plane Assembly with dedicated Proximity Electronics to pilot detector in terms of integration time, windowing and binning;

- optical Module in which the optical elements, described in previous paragraph, are kept in stable position by means of a dedicated optical bench able to guarantee the optical 


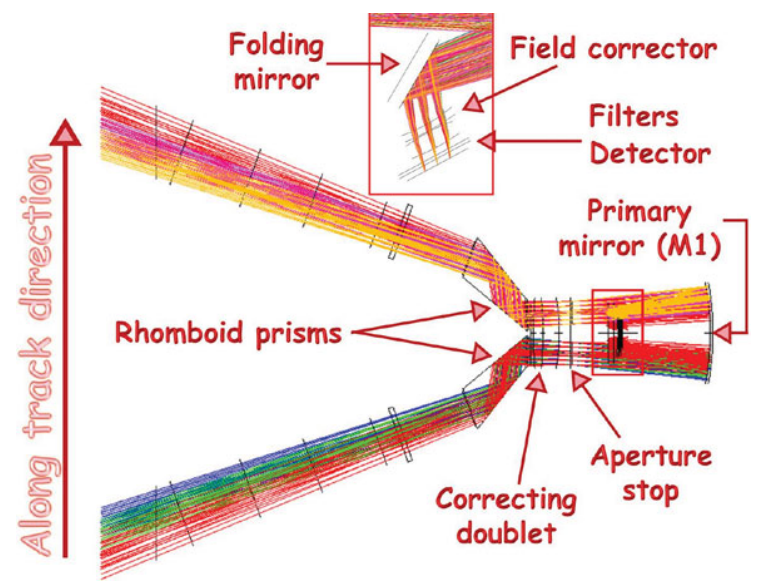

Figure 3. Schematic of the STC optical design

stability after launch, whose random vibration level are very demanding, and within the operative temperature range that is $-20 \div+30{ }^{\circ} \mathrm{C}$;

- external and internal baffling system.

Considering the extreme thermal environment of Mercury whose solar constant is up to $144000 \mathrm{~W} / \mathrm{m}^{2}$, and surface temperature of Planet varies from about $100 \mathrm{~K}$ (dark side) to $690 \mathrm{~K}$ (illuminated side), the thermal architecture is conceived in order to kept thermally decoupled the 3 sub-units from the Planet environment and the spacecraft $(\mathrm{S} / \mathrm{C})$. This is the reason why the external baffling is connected to a dedicated interface provided by S/C, while the STC Optical Bench and FPA with its PE, are connected to a high thermal resistance structure (shared with VIHI) made of sandwich panels with aluminum honeycomb and CFRP skins. To minimize the heat flux coming inside the telescope from the scene a heat rejection filter could be positioned on the honeycomb structure, in between the telescope entrances and the external baffles (Figure 4). The thermal architecture is completed by two Heat Pipes, provided by the S/C, reaching a maximum temperature of about $+16{ }^{\circ} \mathrm{C}$ in hot case, to dissipate the environmental and internally generated heat powers, and by thermal straps accurately designed to cold down respectively the $\mathrm{PE}$ and the detector. In this way the maximum operative temperature of $\mathrm{PE}$ is limited at about $40{ }^{\circ} \mathrm{C}$, while the Detector maximum temperature at about $0{ }^{\circ} \mathrm{C}$ (Begin of Life) thanks to a dedicated Thermo Electric Cooler (TEC) Module. In particular the TEC module and the required electrical power are sized to provide margins on the minimum temperature on detector side. In fact detector sensitivity may change from the BoL up to the EoL mainly due to the interaction with the high energy particles: the possibility to cold down the detector up to $-5{ }^{\circ} \mathrm{C}$ will allow the recovery of its performances. 


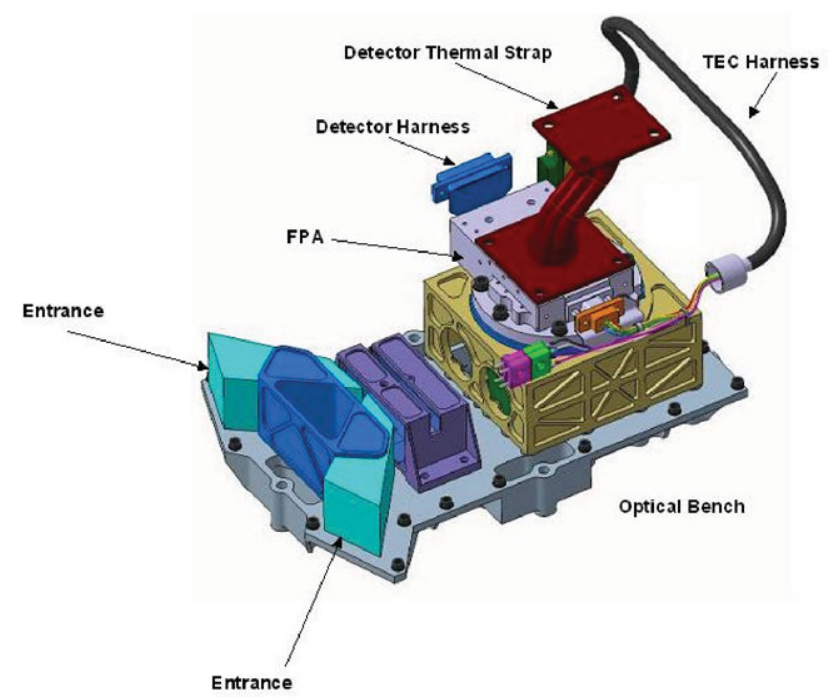

Figure 4. The optical bench of STC

\section{Acknowledgement}

\section{References}

Cremonese, G., Fantinel, D., Giro, G., Capria, M. T., Da Deppo, V., Naletto, G., Forlani, G., Massironi, M., Giacomini, L., Sgavetti, M., Simioni, E., Bettanini, C., Debei, S., Zaccariotto, M., Borin, P., Marinangeli, L., \& Flamini, E. 2009, The stereo camera on the BepiColombo ESA/JAXA mission: a novel approach, Advances in Geosciences, 15, 305.

Massironi, M., Giacomini, L., Cremonese, G., Capria, M. T .,Da Deppo, V. , Forlani, G., Naletto, G., Pasquar, G., \& Flamini, E. 2008, Simulations using terrestrial geological analogues of the Hermean surface to examine 3D rendering potentialities of the STereoscopic imaging Channel of the SIMBIO-SYS package (Bepi-Colombo mission), Plan.Space Scie., 56, 1079

Flamini, E., Capaccioni, F., Colangeli. L., Cremonese, G., Doressoundiram, A., Josset, J. L., Langevin, Y., Debei, S., Capria, M. T., DeSanctis, M. C., Marinangeli, L., Massironi, M., Mazzotta Epifani, E., Naletto, G., Palumbo, P., Eng, P., Roig, J. F., Caporali, A., DaDeppo, V., Erard, S., Federico, C., Forni, O., Sgavetti, M., Filacchione, G., Giacomini, L., Marra, G.,Martellato, E., Zusi, M., Cosi, M., Bettanini, C., Calamai, L., Zaccariotto, M., Tommasi, L., Dami, M., Ficai Veltroni, I., Poulet, F., Hello, Y., and the SIMBIO-SYS Team, 2010 SIMBIO-SYS: the Spectrometers and Imagers Integrated Observatory SYStem for BepiColombo Orbiter, Plan.Space Sci., 58, 125. 